\title{
El desplazamiento activo al Centro educativo influye en el rendimiento académico de las adolescentes españolas \\ Active commuting to school influences on academic performance of Spanish adolescent girls Alberto Ruiz-Ariza, Manuel J.Dela Torre-Cruz, Sara Suárez-Manzano, Emilio J.Martínez-López Universidad de Jaén (España)
}

\begin{abstract}
Resumen. El desplazamiento activo se define como la acción de dirigirse al Centro educativo por medio de transportes que conlleven gasto metabólico, como andar o usar la bicicleta. El tiempo medio diario de desplazamiento activo en adolescentes es de 18 minutos, y podría incrementar un $13 \%$ la actividad física total diaria. El desplazamiento activo se ha asociado a una mejor salud y rendimiento cognitivo. Sin embargo, los estudios que analizan la asociación entre desplazamiento activo y rendimiento académico son muy escasos. El objetivo fue analizar el grado de asociación del desplazamiento activo con el rendimiento académico en Matemáticas, Lengua, Educación Física y con el rendimiento académico general -promedio de Matemáticas, Lengua y Educación Física- en adolescentes. En el presente estudio transversal participaron 1.006 adolescentes españoles. El modo de desplazamiento se evaluó mediante cuestionario y para el rendimiento académico se solicitó a los institutos las calificaciones numéricas. Los adolescentes se clasificaron en inactivos (empleaban transporte motorizado o realizaban menos de cinco trayectos/semana andando durante más de 15 minutos), y activos (realizaban e» cinco trayectos/semana andando más de 15 minutos). Se realizaron análisis de regresión lineal y ANCOVA ajustados por edad e Índice de Masa Corporal. Los resultados mostraron que las chicas que realizan un mayor número de desplazamientos activos semanales tenían un mayor rendimiento académico en Matemáticas ( $\beta=0,041 \pm 0,019, p=0,036)$, en Educación Física $(\beta=0,029 \pm 0,013$, $p=0,029)$, y mayor rendimiento académico general $(\beta=0,031 \pm 0,014, p=0,027)$. En chicos, ninguna asociación resultó significativa (todos $\mathrm{p}>0,05)$. Se concluye que desplazarse al instituto andando, al menos cinco trayectos semanales de más de 15 minutos, se relaciona con mayores calificaciones en Matemáticas en las chicas adolescentes. Fomentar el desplazamiento activo, desde el ámbito escolar y familiar puede ser potencialmente relevante para mejorar el rendimiento académico en chicas españolas de Educación Secundaria.
\end{abstract}

Palabras clave: Caminar, asignaturas, cognición, educación, rendimiento cognitivo.

\begin{abstract}
Active commuting is defined as the action of going to school using a means of transportation that implies energy consumption, such as walking or cycling. Daily average time of active commuting in adolescents is 18 minutes, and this time could increase the total daily physical activity account by $13 \%$. Active commuting has been associated with better health and cognitive performance. However, studies examining the association between active commuting and academic performance are scarce. Based on the above statements, the aim was to analyse the association between active commuting to school and academic performance in Maths, Spanish Language, Physical Education and general academic performance -mean of Math, Language and Physical Education - in adolescents. 1,006 Spanish youths participated in the present cross-sectional study. We used a weekly questionnaire to assess active commuting, whereas we requested academic marks in order to track academic performance. Adolescents were classified as inactive (using passive transport or walking 15' or more less than five times a week) and active (walking 15' or more at least six times a week). Linear regression and ANCOVA analyses adjusted by age and body mass index were performed. Our results showed that girls who carry out a higher number of weekly active trips to school had higher Maths and Physical Education marks ( $\beta=0,041 \pm 0,019, p=0,036 ; \beta=0,029 \pm 0,013, p=0,029$, respectively), and better general academic performance ( $\beta=0,031 \pm 0,014, \mathrm{p}=0,027)$. There were no associations in boys (all $\mathrm{p}>0,05)$. Commuting actively to school for more than 15' a day at least five times a week is associated with better Math performance in adolescent girls. Promotion of active commuting from both school staff and families could be potentially relevant to improve academic performance in Spanish Secondary school girls.
\end{abstract}

Key words: Walking, subjects, cognition, education, cognitive performance.

\section{Introducción}

La práctica habitual de actividad física (AF) se asocia con una mejora muscular, ósea, y cardiovascular (Gracia-Marco et al., 2011), contribuyendo además a un mayor nivel de bienestar psicológico (PadillaMoledo et al., 2012). En jóvenes, las recomendaciones actuales sobre estilo de vida saludable aconsejan realizar al menos 60 minutos diarios de AF a una intensidad de moderada a vigorosa (Oja, Bull, Fogelholm, y Martin, 2010). Sin embargo, la mayoría de niños no alcanza estos mínimos, y durante la adolescencia los minutos de práctica de $\mathrm{AF}$ saludable disminuyen dramáticamente (Chillón et al., 2009; Ramos, Jiménez-Iglesias, Rivera, y Moreno, 2016). Algunos autores han sugerido que fomentar el desplazamiento activo al colegio podría ayudar a compensar este déficit de AF diaria (Chillón et al., 2011; Segura-Díaz, Herrador-Colmenero, Martínez-Téllez, y Chillón, 2015; Villa-González, Ruiz, y Chillón, 2016) y contrarrestar, en parte, los nocivos efectos derivados de los actuales estilos de vida sedentarios (Aires et al., 2011).

El desplazamiento activo se define como la acción de dirigirse al Centro educativo por medio de transportes que conlleven gasto metabólico tales como andar o usar la bicicleta (Chillón et al., 2011; Mandic et al., 2015; Segura-Díaz et al., 2015). Mendoza et al. (2011), concluyeron que el tiempo medio diario empleado en el desplazamiento activo al Centro educativo en adolescentes estadounidenses es de 18 minutos, y podría incrementar un $13 \%$ la AF total practicada durante el

Fecha recepción: 28-07-16. Fecha de aceptación: 25-10-16 Alberto Ruiz Ariza alberto_ruyz@hotmail.com día (Smith et al., 2012). En otro estudio con adolescentes holandeses, Van Dijk, De Groot, Van Acker, Savelberg, y Kirschner (2014), hallaron que el desplazamiento activo constituía el 28\% del total de práctica de AF semanal. Se ha comprobado, que los jóvenes que se desplazan de forma activa al Centro educativo presentan niveles más bajos de sobrepeso y obesidad (Bere, Oenema, Prins, Seiler, y Brug, 2011), mayor felicidad y bienestar (Ruiz-Ariza, De la Torre-Cruz, RedecillasPeiró, y Martínez-López, 2015), y un mayor nivel de condición física (Chillón et al., 2011; Villa-González, Ruiz, y Chillón, 2015). Sin embargo, la asociación entre el desplazamiento activo y parámetros relacionados con la cognición en adolescentes ha sido poco estudiada y las relaciones no son tan evidentes (Martínez-Gómez et al., 2011; Van Dijk et al., 2014).

Algunos autores han informado que la práctica de $\mathrm{AF}$ produce beneficios a nivel cognitivo tanto si se realiza a alta (Ardoy et al., 2014) como a moderada intensidad(Backes, Horvath, y Kazial, 2015; MartínezGómez et al., 2011). La AF mejora la circulación sanguínea cerebral y la plasticidad sináptica (Hillman, Erickson, y Kramer, 2008), ayuda a la supervivencia de las neuronas y fomenta su crecimiento (Ardoy et al., 2014). Estas adaptaciones fisiológicas mejoran tanto la memoria como la capacidad de aprendizaje (Wrann et al., 2013), y en definitiva la función cognitiva, uno de los factores considerados clave en el rendimiento académico (RA) (Hillman et al., 2009; Mora-Corral, 2010).

El RA hace referencia al éxito de un alumno en el Centro Educativo medido a partir de las calificaciones en las diferentes asignaturas (RuizAriza, Ruiz, de la Torre-Cruz, Latorre-Román, y Martínez-López, 2016). Se ha comprobado que el RA puede estar mediatizado por la valoración de los profesores hacia las actitudes de los alumnos, así como 
el interés, la participación y la asistencia a clase de los mismos (Gutiérrez y López, 2012). Las estrategias de autoeficacia y autorregulación del aprendizaje, las estrategias de apoyo, de organización y comprensión, así como las metas de aprendizaje, también contribuyen a la explicación del RA(Valle et al., 2009). Además, puede estar influenciado por otras variables deíndole afectivo-emocional (Cueli, González-Castro, Álvarez, García, y González-Pienda, 2014) o socioeconómico (Castillo et al., 2011). Sin embargo, para algunos autores, la capacidad cognitiva es uno de los factores más determinantes en el éxito académico (Laidra, Pullman, y Allik, 2007; Mora-Corral, 2010).

Romeo y McEwen (2006) sugirieron la necesidad de una mayor estimulación cognitiva durante la adolescencia debido a que aún se mantiene un alto grado de plasticidad cerebral, indispensable para poder lograr aprendizajes significativos. Dicha estimulación es determinante para la mejora del RA y podría ser favorecida por la AF diaria y sistemática que implica el desplazamiento activo al instituto (Martínez-Gómez et al., 2011; Stock et al., 2012). De hecho, aparte de la Educación Física (EF) escolar, el RA mejoraría significativamente a partir de 17 minutos de ejercicio de moderado a vigoroso al día en chicos y 12 minutos en chicas (Booth et al., 2014).

Martínez-Gómez et al. (2011), hallaron que las adolescentes españolas que realizaban desplazamiento activo al instituto tenían mejores índices de rendimiento cognitivo, medido a través de un cuestionario estandarizado de inteligencia y habilidades básicas de aprendizaje. Stock et al. (2012), en una muestra de 10380 adolescentes daneses, comprobaron que los jóvenes con una percepción más alta de su RA se desplazaban de forma activa en mayor medida que aquellos con una percepción más baja. No obstante, los estudios que analizan la asociación del desplazamiento activo al Centro educativo con el RA son muy escasos (Domazet et al., 2016; Haapala et al., 2014). Van Dijk et al., (2014), han asociado recientemente el desplazamiento activo y el RA en adolescentes, analizando las posibles diferencias en función del sexo. Estos autores hallaron una asociación muy débil entre ambas variables, encontrando solo relación entre el desplazamiento activo y los niveles de atención mediante el test d2 en chicas. Este último estudio es muy relevante debido a que fue llevado a cabo en Holanda, una población con características de desarrollo similares a la española, pero que sin embargo se encuentra entre los principales países del mundo con mayor tasa de éxito escolar(PISA, 2012).

En base a lo expuesto anteriormente, el objetivo del presente estudio fue analizar la asociación entre el desplazamiento activo al instituto y las calificaciones en las asignaturas de Matemáticas, Lengua y EF en una muestra de jóvenes españoles.

\section{Metodología}

\section{Diseñoy participantes}

Se trata de un estudio transversal cuantitativo. Se empleó como covariables la edad y el IMC de los adolescentes. Durante el curso académico 2013-2014 se tomó una muestra inicial de 1224 adolescentes, pertenecientes a 12 institutos de Educación Secundaria de la provincia de Jaén (España) (7 urbanos e»10000 habitantes, y 5 rurales $<10000$ habitantes). Fueron excluidos aquellos alumnos que contestaron de forma incompleta o no mostraron autorización parental. Se excluyeron del estudio aquellos participantes que manifestaron emplear la bicicleta como medio de transporte activo, debido a que el número de alumnos que usaban este medio era insignificante. La muestra final de estudio estuvo formada por un total de 1006 estudiantes de Educación Secundaria.

\section{Instrumentos}

\section{Rendimiento Académico}

La calificación en las asignaturas de Matemáticas y Lengua -como variables con mayor poder explicativo de RA(Miñano, Gilary Castejón, 2012; Ruiz-Ariza et al., 2016) - y las notas de EF — como referente de la actitud del niño hacia la AF-, fueron las variables dependientes analizadas en este estudio. Se solicitó a los Centros Educativos las calificaciones correspondientes a la evaluación trimestral previa a la cumplimentación del cuestionario (escala 1-10). Para obtener la medida de RA general se realizó un promedio de las calificaciones de las tres asignaturas.

Cuestionario de modo de desplazamiento al instituto

Se evaluó mediante un cuestionario que registró el modo, tiempo, y cantidad de desplazamientos semanales empleados para ir y volver del Centro educativo (Chillón et al., 2009; 2011). La variable independiente fue el número de trayectos de ida o vuelta semanales que cada alumno realizaba andando durante un tiempo superior a 15 minutos. Los adolescentes se clasificaron en inactivos y activos: inactivos (empleaban transporte motorizado o realizaban menos de cinco trayectos/semana andando) y activos (realizaban e» cinco trayectos andando - superiores a 15 minutos-durante la semana). Los valores oscilaban entre cero y 10 trayectos. Se tomó la cifra de 15 minutos en base a lo establecido en los estudios de Martínez-Gómez et al. (2011) y Ruiz-Ariza et al. (2015) [Figura 1].

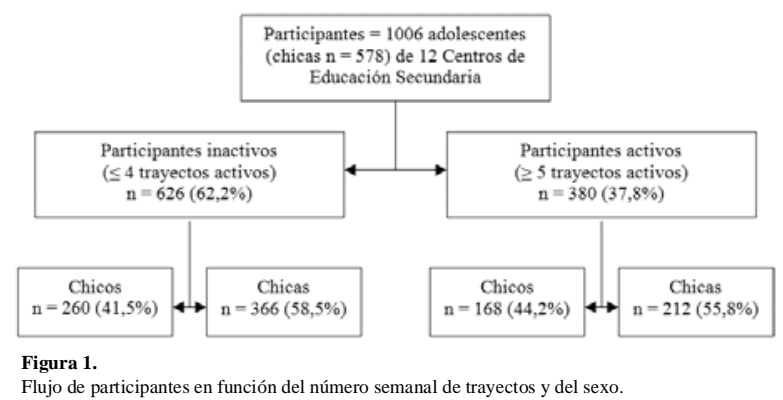

\section{Covariables}

Se consideró como variable de confusión la edad debido a que está inversamente relacionada con la práctica de AF y con el RA durante la adolescencia (Esteban-Cornejo, Tejero-Gonzalez, Sallis, y Veiga, 2015; Ruiz-Ariza et al., 2016). Muchos estudios han considerado también que el IMC ejerce una alta influencia tanto en la cantidad de práctica de AF como en el RA durante la adolescencia (Ruiz-Ariza et al., 2016; Sardinha et al., 2014). El IMC se calculó mediante la fórmula = peso (kg) / talla $\left(\mathrm{m}^{2}\right)$. Para obtener las medidas de peso y talla se empleó una báscula digital ASIMED modelo Elegant ${ }^{\circledR}$ tipo B - clase III-, y un tallímetro portátil SECA® 214 (SECA Ltd., Hamburgo, Alemania), respectivamente. Ambas medidas se realizaron descalzos y con ropa ligera.

\section{Procedimiento}

Se contactó vía correo postal con los Centros educativos y se contó con la autorización de los directores y profesores de EF. Se informó a padres y tutores legales de los alumnos sobre la naturaleza y el propósito del estudio. Todos dieron su consentimiento escrito para la participación de sus hijos en este estudio. El nombre de cada participante se codificó para asegurar el anonimato y confidencialidad de las respuestas emitidas. Varios investigadores debidamente formados se desplazaron a los Centros educativos. El registro de datos se llevó a cabo dentro de la clase de Educación Física. Un cuestionario sociodemográfico de datos personales como la edad y el sexo, y el cuestionario sobre desplazamiento activo al Centro educativo fueron administrados de forma individual. La duración de la toma de datos fue de 40 minutos.

\section{Normativa legal y comité de bioética}

El estudio fue aprobado por la Comisión de Bioética de la Universidad de Jaén. En el diseño se han tenido en cuenta la normativa legal vigente española que regula la investigación clínica en humanos (Real Decreto 561/1993 sobre ensayos clínicos), la ley de protección de datos de carácter personal (Ley Orgánica 15/1999), así como los principios fundamentales establecidos en la Declaración de Helsinki (revisión de 2013). 


\section{Análisis estadístico}

Los parámetros se muestran como media, desviación típica y error estándar. Las diferencias descriptivas entre sexo se analizaron mediante la prueba t de Student para variables continuas y el test $\div$ para variables categóricas. Se realizaron análisis de regresión lineal para estudiar la asociación del número de transportes semanales considerados activos (variable independiente) con el RA en las diferentes asignaturas (variables dependientes). Además, para estudiar si los adolescentes considerados activos tenían a su vez un mejor RA que los jóvenes inactivos se realizó análisis de covarianza ANCOVA. Se introdujeron las calificaciones de cada asignatura como variables dependientes y la clasificación dicotomizada en función del número de trayectos de más de 15 minutos (activos vs. no activos) como factor fijo. La muestra fue segmentada por sexo para todos los análisis debido a que previamente se había hallado interacción entre sexos y medidas de $\operatorname{RA}(p<0,05)$. Todos los análisis se realizaron separadamente para cada asignatura y se ajustaron por edad e IMC, ya que se halló correlación entre edad e IMC con respecto al RA (ambas $p<0,01$ ). Para todos los análisis se empleó un nivel de confianza del 95\%. Los cálculos se realizaron con el programa estadístico SPSS, v. 19,0 para WINDOWS (SPSS Inc., Chicago).

\begin{tabular}{|c|c|c|c|c|c|c|c|}
\hline & $\begin{array}{r}\text { Todos } \\
(\mathrm{n}=10 \mathrm{C}\end{array}$ & & $\begin{array}{r}\text { Chico } \\
(\mathrm{n}=42 \\
\end{array}$ & & $\begin{array}{c}\text { Chicas } \\
(\mathrm{n}=578)\end{array}$ & & \\
\hline & Media I & $\mathrm{DT}$ & Media & DT & Media D & DT & $P$ \\
\hline Edad (años) & 14,43 & 1,69 & 14,47 & 1,74 & 14,39 & 1,66 & $5 \quad 0,431$ \\
\hline Peso $(\mathrm{kg})$ & 59,09 & 13,37 & 63,22 & 14,9 & 56,04 & 11,19 & $<0,001$ \\
\hline Talla (m) & 1,64 & 0,09 & 1,69 & 0,09 & 1,61 & 0,07 & $<0,001$ \\
\hline IMC $\left(\mathrm{kg} / \mathrm{m}^{2}\right)$ & 21,80 & 3,92 & 22,01 & 4,12 & 21,65 & 3,75 & 0,149 \\
\hline RA en Matemáticas & 6,16 & 2,14 & $4 \quad 5,98$ & 2,20 & 6,29 & 2,08 & 0,023 \\
\hline RA en Lengua & 6,33 & 1,97 & 5,87 & 1,99 & 6,67 & 1,88 & $<0,001$ \\
\hline RA en Educación Física & 7,44 & 1,48 & $3 \quad 7,36$ & 1,53 & 7,51 & 1,43 & 0,117 \\
\hline RA General: $($ Mat+Len+EF)/3 & 6,64 & 1,54 & $4 \quad 6,40$ & 1,55 & 6,82 & 1,52 & $<0,001$ \\
\hline $\begin{array}{l}\text { Tiempo promedio de desplazamiento } \\
\text { activo (minutos/día) }\end{array}$ & 18,30 & 1,14 & 18,45 & 1,11 & 18,25 & 1,16 & 0,620 \\
\hline $\begin{array}{l}\text { Trayectos semanales totales }>15 \\
\text { minutos }\end{array}$ & 5,74 & 4,50 & 5,69 & 4,47 & 5,78 & 4,53 & 0,764 \\
\hline $\begin{array}{l}\text { Trayectos de > } 15 \text { minutos, } 5 \text { o más } \\
\text { veces/semana }(\mathrm{n}(\%))\end{array}$ & $380(37,8$ & & $168(39$ & & $212(36,7)$ & & 0,913 \\
\hline
\end{tabular}

\begin{tabular}{|c|c|c|c|c|c|c|c|c|}
\hline & \multicolumn{5}{|c|}{ Chicos (428) } & \multicolumn{3}{|c|}{ Chicas (578) } \\
\hline & & & & Mate & máticas & & & \\
\hline & $\beta$ & $\mathrm{EE}$ & $\mathrm{r}$ & & $\beta$ & $\mathrm{EE}$ & $\mathrm{r}$ & $\mathrm{P}$ \\
\hline$\overline{\text { Edad }}$ & $-0,249$ & 0,063 & $-0,195$ & $<0,001$ & $-0,113$ & 0,053 & $-0,089$ & 0,035 \\
\hline IMC & $-0,020$ & 0,026 & $-0,038$ & 0,437 & $-0,033$ & 0,023 & $-0,061$ & 0,151 \\
\hline \multirow[t]{3}{*}{ Trayectos semanales activos } & 0,011 & 0,024 & 0,022 & 0,642 & 0,041 & 0,019 & 0,089 & 0,036 \\
\hline & \multicolumn{8}{|c|}{ Lengua } \\
\hline & $\beta$ & $\mathrm{EE}$ & $\mathrm{r}$ & $\mathrm{P}$ & $\beta$ & $\mathrm{EE}$ & $\mathrm{r}$ & $\mathrm{P}$ \\
\hline$\overline{\text { Edad }}$ & $-0,185$ & 0,057 & $-0,159$ & 0,001 & $-0,102$ & 0,048 & $-0,089$ & 0,036 \\
\hline IMC & $-0,031$ & 0,024 & $-0,064$ & 0,193 & $-0,036$ & 0,021 & $-0,072$ & 0,087 \\
\hline \multirow[t]{3}{*}{ Trayectos semanales activos } & 0,006 & 0,022 & 0,014 & 0,767 & 0,024 & 0,018 & 0,057 & 0,179 \\
\hline & \multicolumn{8}{|c|}{ Educación Física } \\
\hline & $\beta$ & $\mathrm{EE}$ & $\mathrm{r}$ & $\mathrm{P}$ & $\beta$ & $\mathrm{EE}$ & $\mathrm{r}$ & $\mathrm{P}$ \\
\hline Edad & $-0,028$ & 0,044 & $-0,032$ & 0,521 & $-0,008$ & 0,037 & $-0,009$ & 0,824 \\
\hline IMC & $-0,052$ & 0,018 & $-0,142$ & 0,004 & $-0,049$ & 0,016 & $-0,129$ & 0,002 \\
\hline \multirow[t]{3}{*}{ Trayectos semanales activos } & 0,003 & 0,017 & $.0,008$ & 0,872 & 0,029 & 0,013 & 0,092 & 0,029 \\
\hline & \multicolumn{8}{|c|}{ Rendimiento Académico General } \\
\hline & $\beta$ & $\mathrm{EE}$ & $\mathrm{r}$ & $\mathrm{P}$ & $\beta$ & $\mathrm{EE}$ & $\mathrm{r}$ & $\mathrm{P}$ \\
\hline Edad & $-0,154$ & 0,044 & $-0,171$ & 0,001 & $-0,074$ & 0,039 & $-0,080$ & 0,057 \\
\hline IMC & $-0,034$ & 0,018 & $-0,092$ & 0,060 & $-0,039$ & 0,017 & $-0,098$ & 0,020 \\
\hline Trayectos semanales activos & 0,007 & 0,017 & 0,019 & 0,687 & 0,031 & 0,014 & 0,093 & 0,027 \\
\hline
\end{tabular}

\section{Resultados}

Los participantes tenían una edad promedio de 14,43 $\pm 1,69$ años (rango: 12-17 años) y un IMC de 21,80 $\pm 3,92 \mathrm{~kg} / \mathrm{m}^{2}$. El 42,5\% fueron chicos $(n=428)$ y el $57,5 \%$ chicas $(n=578)$. El 37,7\% de los 1006 participantes realizaba 5 o más trayectos semanales de más de 15 minutos. Las características sociométricas, nivel de RA, y desplazamiento activo se presentan en la Tabla 1 . Los resultados mostraron que las chicas tenían mejores calificaciones en Matemáticas $(p=0,023)$ y en Lengua ( $p<0,001)$, y un mayor RA general que los chicos $(p<0,001)$. El número de trayectos y el tiempo promedio empleado durante la semana fue similar en chicos y chicas $(p>0,05)$ [ver tabla 1 ].
Los resultados del análisis de regresión lineal que muestra la asociación entre el número de desplazamientos activos al instituto (trayectos andando superiores a 15 minutos)y el RA en Matemáticas, Lengua, EF, y RA general se presentan en la tabla 2. Las chicas que realizaban más trayectos activos semanales tenían significativamente mejores calificaciones en Matemáticas ( $\beta=0,041 \pm 0,019, p=0,036)$, y EF ( $\beta=0,029$ $\pm 0,013, p=0,029)$, así como un mayor RA general $(\beta=0,031 \pm 0,014$; $p=0,027)$. Sin embargo, las anteriores asociaciones no se encontraron en los chicos (todos $p>0,05$ ). En la asignatura de Lengua no se halló asociación respecto al número de trayectos activos en ninguno de los dos sexos $(p>0,05)$. Se observó también que la edad se asociaba negativamente con la calificación de Matemáticas $(\beta=-0,249 \pm 0,063$, $p<0,001$ en chicos, $\mathrm{y} \beta=-0,113 \pm 0,053, p=0,035$ en chicas) y Lengua ( $\beta=-0,185 \pm 0,057, p=0,001$ en chicos, $\mathrm{y} \beta=-0,102 \pm 0,048, p=$ 0,036 en chicas), y con el RA general solo en chicos, esto es, a mayor edad menor RA ( $\beta=-0,154 \pm 0,044, p=0,001)$. El IMC se asoció negativamente con la calificación de $\mathrm{EF}$ en ambos sexos $(\beta=-0,052 \pm$ $0,018, p<0,004$ en chicos, $\mathrm{y} \beta=-0,049 \pm 0,016, p=0,002$ en chicas), $\mathrm{y}$ con el RA general en chicas $(\beta=-0,039 \pm 0,017, p=0,020$ ).

Los resultados del análisis ANCOVA que estudia si los adolescentes activos ( $>5$ trayectos semanales de más de 15 minutos) tenían mejor calificación en Matemáticas, Lengua, EF, y RA general que los inactivos (empleaban transporte motorizado o realizaban menos de cinco trayectos/semana andando), se muestran en la figura 2. Los resultados mostraron que las chicas activas obtenían significativamente mejores calificaciones en Matemáticas ( $p=0,027)$ y en EF ( $p=0,005)$, así como un mejor RA general $(p=0,008)$ que las inactivas. Los resultados mostraron también que no existen diferencias entre chicos activos e inactivos en ninguna de las variables estudiadas - Matemáticas, Lengua, EF, y RA general- (todas $p>0,05$ ). En la asignatura de Lengua los resultados de chicos y chicas en función del grado de AF semanal fueron similares $(p>0,05)$
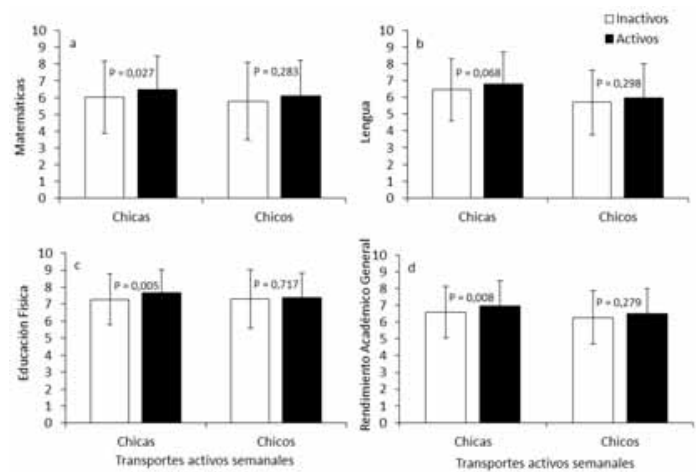

Figura 2. Asociacion entre niveles de transporte al centro educativo (inactivos vs. activos) con la calificación en las asignaturas de Matemáticas (a), Lengua (b), Educación Física (c), y rendimiento académico general (d) en adolescentes españoles de 12 - 17 años. El trasporte escolar se clasificó como inactivos (empleaban transporte motorizado o menos de cinco trayectos andando/semana) vs. activos (= cinco trayectos andando/semana $=15$ minutos). Todos los análisis fueron ajustados por edad e IMC. Resultados expresados como media y desviación estándar.

\section{Discusión}

El presente trabajo ha estudiado la relación del desplazamiento activo al instituto respecto a las calificaciones en las asignaturas de Matemáticas, Lengua y EF, así como en RAgeneral en base al promedio de las tres, en una muestra de jóvenes españoles. Los resultados muestran que los adolescentes activos realizaban un promedio de 18 minutos al día de desplazamiento andando al Centro educativo, sin haber diferencias significativas por sexo. Las chicas que realizan más trayectos activos semanales obtienen significativamente mejores calificaciones en Matemáticas y EF, así como mejor RAgeneral. Sin embargo, en chicos no se han hallado diferencias de RA en función del tipo de desplazamiento al instituto.

Nuestros resultados muestran que las chicas que realizan más trayectos activos semanales obtienen mejores calificaciones en Matemáticas, pero no en Lengua. Estos son similares a los hallados por Martínez- 
Gómez et al. (2011), que mostraron que las chicas adolescentes que invierten más de 15 minutos al día en desplazamiento activo al Centro educativo, presentan niveles más altos de rendimiento cognitivo. Además, mientras algunas investigaciones no han hallado asociación entre desplazamiento activo y RA(Van Dijk et al., 2014), otros autores han concluido que desplazarse de forma activa al instituto se asocia con mejores calificaciones en Matemáticas, pero no incluyen resultados con respecto a Lengua - tiempo de desplazamiento no reportado(Domazet et al., 2016). Esta divergencia entre Matemáticas y Lengua, podría deberse a que una mayor AF influye en la codificación simbólica, mejora los recursos atencionales, y el procesamiento durante las tareas aritméticas (Moore, Drollette, Scudder, Bharij, y Hillman, 2014). Mientras que la influencia de laAF sobre el lenguaje está aún menos explorada (Ardoy et al., 2014).

Nosotros hemos observado que el desplazamiento activo ejerce además un efecto diferencial en función del sexo, ya que afecta al RA en Matemáticas, EF y al RA general en chicas pero no en chicos. Estos resultados son similares a los obtenidos por Martínez-Gómez et al. (2011) y Van Dijk et al. (2014), que hallaron asociación positiva entre el desplazamiento activo al Centro educativo y el rendimiento cognitivo solo en chicas. Algo similar ocurre con los resultados de asociación que Kwak et al. (2009), hallaron entre AF y RA en adolescentes suecas, y con los de Ruiz-Ariza et al. (2016), en adolescentes españolas. Para estos autores el RA podría verse incrementado en chicas por la práctica de AF o por el simple disfrute con esta práctica, respectivamente, pero solo si se llevaba a cabo a una intensidad alta o vigorosa.

Aunque el efecto del ejercicio vigoroso está ampliamente contrastado (Kwak et al., 2009; Ruiz-Ariza et al., 2016; So, 2012), nuestros hallazgos y los obtenidos por Hillman et al. (2009), Martínez-Gómez et al. (2011), Stock et al. (2012), Van Dijk et al. (2014), y Domazet et al. (2016), demuestran que el desplazamiento activo al Centro educativo, a pesar de estar considerado como de intensidad leve o moderada, influye también positivamente sobre el rendimiento cognitivo y RA. Por tanto, la influencia de la intensidad del ejercicio físico sobre el RA es un factor más a estudiar, ya que podría tener efectos distintivos en las funciones cerebrales (Liu et al., 2009; Martínez-Gómez et al., 2011; Reigal, Borrego, Juárez, y Hernández-Mendo, 2016).

Las causas por las que se obtiene diferente efecto sobre el RA en chicos y chicas no pueden ser explicadas a partir del presente estudio. Sin embargo, existen dos posibles razonamientos. Por un lado, el estrés ocasionado por los cambios biológicos y psicológicos de la adolescencia puede provocar problemas de ansiedad o depresión, sobre todo en chicas (Rudolph, 2002). Estos problemas tienen un efecto negativo sobre el RA a corto y largo plazo (Suhrcke y de Paz-Nieves, 2011). Se ha demostrado que la práctica de AF incrementa el factor neurotrófico derivado del cerebro (BDNF) (Ruiz-Ariza, Grao-Cruces, de Loureiro, y Martínez-López, 2016; Wrann et al., 2013), que es determinante en el aumento de las funciones cognitivas (Hillman et al., 2009) y en la prevención de la depresión en chicas (Autry, Adachi, Cheng, y Monteggia, 2009). En este caso, el desplazamiento activo al Centro educativo podría aumentar el BDNF, reducir el estrés, la ansiedad y la depresión, así como mejorar el RA. Por otro lado, el desplazamiento activo podría aumentar la producción y circulación del factor de crecimiento insulínico I (IGF-1). Este IGF-1 interactúa con el estrógeno femenino promoviendo la supervivencia neuronal y la neuroprotección (Van Dijk et al., 2014). Por último, el efecto diferencial del sexo podría encontrarse en la asociación entre las habilidades sociales y el RA. Algunos estudios han informado que el desplazamiento activo al Centro educativo favorece el contacto social entre vecinos y mejora las habilidades sociales (Giles-Corti, Foster, Shilton, y Falconer, 2010). Durlak, Weissberg, Dymnicki, Taylor, y Schellinger (2011), asociaron las anteriores mejoras con un aumento de los resultados académicos. Sin embargo, aunque las chicas podrían mostrar mejores habilidades sociales que los chicos (Garaigordobil y Durá, 2006), no tenemos evidencia de que las chicas del presente estudio se hayan visto afectadas por los beneficios sociales del desplazamiento activo en mayor medida que los chicos, y por tanto, obtengan mejor RA.
El presente estudio presenta un alto grado de similitud con resultados previos que han puesto el foco en distintos tipos de AF en jóvenes y su relación con el rendimiento cognitivo y académico. Haapala et al. (2014), en jóvenes finlandeses, y Ruiz et al. (2010), en adolescentes españoles, mostraron que la AF extraescolar no perjudica al tiempo de estudio y al tiempo dedicado a la realización de tareas escolares, sino que esta AF influye positivamente en el rendimiento cognitivo en adolescentes. Por tanto, realizar un desplazamiento de forma activa al Centro educativo podría contribuir a alcanzar los niveles semanales necesarios de AF para influir positivamente en el RA en los jóvenes.

Existen algunas limitaciones que han de ser reconocidas. Por ejemplo, que al tratarse de un estudio transversal no permite establecer relaciones de causalidad. El cuestionario se encuentra en fase de validación, por lo que aún no podemos afirmar que sea válido y fiable. Las respuestas del mismo son auto informadas por los adolescentes que en algunos casos podrían haber sido contestadas erróneamente de forma deliberada o sin mala intención. Además, no se incluyen otras importantes covariables como el estatus socioeconómico familiar. Aunque estos errores podrían haberse visto reducidos ya que se ha utilizado la codificación para asegurar el anonimato y confidencialidad de los participantes y respuestas emitidas. Otras fortalezas pueden ser la amplia muestra utilizada incluyendo chicos y chicas, y el análisis de la relación entre variables hasta el momento muy poco estudiadas.

\section{Conclusiones}

Se concluye que desplazarse al instituto andando, al menos cinco trayectos semanales superiores a 15 minutos/día, se relaciona con calificaciones más altas en Matemáticas, Educación Física y RA general en chicas adolescentes españolas. Fomentar el desplazamiento activo, especialmente en las chicas, desde el ámbito escolar, y familiar puede ser potencialmente relevante para mejorar el RA.

\section{Referencias}

Aires, L., Pratt, M., Lobelo, F., Santos, R., Santos, M., \& Mota, J. (2011). Associations of Cardiorespiratory Fitness in Children and Adolescents With Physical Activity, Active Commuting to School, and Screen Time. Journal of Physical Activity and Health, 8S198-S205.

Ardoy, D. N., Fernández-Rodríguez, J. M., Jiménez-Pavón, D., Castillo, R., Ruiz, J. R., \& Ortega, F. B. (2014).A Physical Education trial improves adolescents' cognitive performance and academic achievement: the EDUFIT study. Scandinavian Journal of Medicine \& Science in Sports, 24(1), e52-e61.

Autry, A. E., Adachi, M., Cheng, P., \& Monteggia, L. M. (2009). Gender-specific impact of brainderivedneurotrophic factor signaling on stress-induced depressionlike behavior. Biological Psychiatry, 66(1), 84-90.

Backes, T. P., Horvath, P. J., \& Kazial, K.A. (2015). The effects of exercise and two pre-exercise fluid amounts on cognition. Journal of Human Sport and Exercise, 10(2), 615-622.

Bere, E., Oenema, A., Prins R., Seiler, S., \& Brug, J. (2011). Longitudinal associations between cycling to school and weight status. International Journal Pediatric Obesity, 6(3-4), 182-187.

Booth, J. N., Leary, S. D., Joinson, C., Ness, A. R., Tomporowski, P. D., Boyle, J. M., \& Reilly, J. J. (2014). Associations between objectively measured physical activity and academic attainment in adolescents from a UK cohort. British Journal of Sport Medicine, 48(1), 265-270.

Castillo, R., Chillón, P., Díaz, L. E., Jiménez-Pavón, D., Moreno, L. A., Ortega, F. B., \& Ruiz, J. R. (2011). Associations between parental educational/ occupational levels and cognitive performance in Spanish adolescents: The AVENAstudy. Psicothema, 23(3), 349-355.

Chillón, P., Gottrand, F., Ortega, F. B., González-Gross, M., Ruiz, J. R., Ward, D. S., ... Molnar, D. (2011). Active Commuting and Physical Activity in Adolescents From Europe: Results From the HELENAStudy.Pediatric Exercise Science, 23(2), 207-217.

Chillón, P., Ortega, F., Ruiz, J., Pérez, I., Martín-Matillas, M., Valtueña, J.,... Delgado, M. (2009). Socio-economic factors and active commuting to school in urban Spanish adolescents: the AVENAstudy. European Journal of Public Health, 19(5), 470-476.

Cueli, M., González-Castro, P., Álvarez, L., García, T., \& González-Pienda, J. A. (2014). Variables afectivo-motivacionales y rendimiento en matemáticas: Un análisis bidireccional. Revista Mexicana de Psicología, 31(2), 153-163.

Domazet, S. L., Tarp, J., Huang, T., Gejl, A. K., Andersen, L. B., Froberg, K., \& 
Bugge, A. (2016). Associations of Physical Activity, Sports Participation and Active Commuting on Mathematic Performance and Inhibitory Control in Adolescents. PloS One, 11(1), e0146319.

Durlak, J., Weissberg, R., Dymnicki,A., Taylor, R., \& Schellinger, K.(2011). The Impact of Enhancing Students'Social and Emotional Leaming:AMeta-Analysis of School-Based Universal Interventions. Child Development, 81(1), 405-432.

Esteban-Cornejo, I., Tejero-Gonzalez, C. M., Sallis, J. F., \& Veiga, O. L. (2015) Physical activity and cognition in adolescents: Asystematic review. Journal of Science and Medicine in Sport/Sports Medicine Australia, 18(5), 534-9.

Garaigordobil, M., \& Durá,A. (2006). Relaciones del autoconcepto y la autoestima con la sociabilidad, estabilidad emocional y responsabilidad en adolescentes de 14-17 años. Análisis y modificación de conducta, 32(141), 37-64.

Giles-Corti, B., Foster, S., Shilton, T., \& Falconer, R. (2010). The co-benefits for health of investing in active transportation. NSW Public Health Bull, 21(5-6), 122-7.

Gracia-Marco, L. L., Vicente-Rodríguez, G. G., Casajús, J. J., Molnar, D. D., Castillo, M. M., \& Moreno, L. L. (2011). Effect of fitness and physical activity on bone mass in adolescents: the HELENA Study. European Journal ff Applied Physiology, 111(11), 2671-2680.

Gutiérrez, M., \& López, E. (2012). Motivación, Comportamiento de los alumnos y rendimiento académico. Infanciay Aprendizaje, 35(1), 61-72.

Haapala, E., Poikkeus, A., Kukkonen-Harjula, K., Tompuri, T., Lintu, N., Väistö, J.,... Lakka, T.(2014). Associations of Physical Activity and Sedentary Behavior with Academic Skills-AFollow-Up Study among PrimarySchool Children. PLOS ONE, 9(9), e107031.

Hillman, C. H., Erickson, K. I., \& Kramer, A. F. (2008). Be smart, exercise your heart: exercise effects on brain and cognition. Nature Reviews Neuroscience, 9(1), 58-65.

Hillman, C. H., Pontifex, M. B., Raine, L. B., Castelli, D. M., Hall, E. E., \& Kramer,A. F.(2009). The effect of acute treadmill walking on cognitive control and academic achievement in preadolescent children. Neuroscience, 159(3), 1044-1054.

Kwak, L., Kremers, S. P., Bergman, P., Ruiz, J. R., Rizzo, N. S., \& Sjostrom, M (2009).Associations between physical activity, fitness, and academic achievement Journal of Pediatric, 155(6), 914-918.

Laidra, K., Pullman, H., \& Allik, J. (2007). Personality and intelligence as predictors of academic achievement:Across-sectional study from elementary to secondary school. Personality and Individual Differences, 42(3), 441-451.

Liu, Y. F., Chen, H. I., Wu, C. L., Kuo, Y. M., Yu, L., Huang, A. M.,... Jen C. J. (2009). Differential effects of treadmill running and wheel running on spatia or aversive learning and memory: roles of amygdalar brainderived neurotrophic factor and synaptotagmin I. Journal of Physiology, 587(13), 3221-3231.

Mandic, S., Mountfort, A., Hopkins, D., Flaherty, C., Williams, J., Brook, E., .. Moore,A. (2015). BuiltEnvironment and Active Transport to School(BEATS) Study: Multidisciplinary and Multi-Sector Collaboration for Physical Activity Promotion (El estudio «Entorno construido y desplazamiento activo a la escuela(BEATS)»: colaboración multidisciplinaria. Retos, 0(28), 197-202.

Martínez-Gómez, D., Ruiz, J. R., Gómez-Martínez, S., Chillón, P., Rey-López, J., Díaz, L. E.,....Marcos, A. (2011). Active Commuting to School and Cognitive Performance in Adolescents: The AVENA Study. Archives of Pediatrics \& Adolescent Medicine, 165(4), 300-305.

Mendoza, J., Watson, K., Nguyen, N., Cerin, E., Baranowski, T., \& Nicklas, T. (2011).Active commuting to school and association with physical activity and adiposity among US youth. Journal of Physical Activity and Health, 8, 488495.

Ministerio de Educación, Cultura y Deporte(2013). PISA2012. Programa para la Evaluación Internacional de los Alumnos OCDE. Informe español. Madrid, España: Ministerio de Educación, Cultura y Deporte.

Miñano, P., Gilar, R., \& Castejón, J. (2012). Un modelo estructural de variables cognitivo-motivacionales explicativas del rendimiento académico en Lengua Española y Matemáticas. Anales De Psicología, 28(1), 45-54.

Moore, R. D., Drollette, E. S., Scudder, M. R., Bharij, A., \& Hillman, C. H. (2014). The influence of cardiorespiratory fitness on strategic, behavioral, and electrophysiological indices of arithmetic cognition in preadolescent children. Frontiers in Human Neuroscience, 8, 258.

Mora-Corral,A. J.(2010). Determinantes del abandono escolar en Cataluña: Más allá del nivel socioeconómico de las familias. Revista de Educación, $n^{\circ}$ extraordinario, 171-190.

Oja, P., Bull, F. C., Fogelholm, M., \& Martin, B. W. (2010). Physical activity recommendations for health: what should Europe do? BMC Public Health, 10(10)

Padilla-Moledo, C., Castro-Piñero, J., Ortega, F. B., Mora, J., Márquez, S., Sjöström, M., \& Ruiz, J. R. (2012). Positive health, cardiorespiratory fitness and fatness in children and adolescents. The European Journal of Public Health, 22(1), 52-56.

Ramos, P., Jiménez-Iglesias, A., Rivera, F., \& Moreno, C. (2016). Physical Activity Trends in Spanish Adolescents. Revista Internacional de Medicina Y Ciencias de La Actividad Física Y Del Deporte, 62(2016), 335-353.

Reigal, R. E., Borrego, J. L., Juárez, R., \& Hernández-Mendo, A. (2016). Práctica física regular y funcionamiento cognitivo en una muestra adolescente. Revista iberoamericana de psicología del ejercicio y el deporte, 2(11), 201-209.

Romeo, R. D., \& McEwen, B. S. (2006). Stress and the adolescent brain. Annals of the New York Academy of Sciences, 1094, 202-214.

Ruiz-Ariza, A., de la Torre-Cruz, M. J., Redecillas-Peiró, M. T., \& Martínez-López, E. J. (2015). Influence of active commuting on happiness, well-being, psychological distress and body shape in adolescents. Gaceta sanitaria, 29(6), 454-457.

Ruiz-Ariza, A., Grao-Cruces, A., de Loureiro, N. E. M., \& Martínez-López, E. J. (2016). Influence of physical fitness on cognitive and academic performance in adolescents: A systematic review from 2005-2015. International Review of Sport and Exercise Psychology, 10(1), 108-133.

Ruiz-Ariza, A., Ruiz, J., De la Torre-Cruz, M., Latorre-Román, P., \& MartínezLópez, E. J. (2016). Influence of level of attraction to physical activity on academic performance of adolescents. Revista Latinoamericana de Psicologia, 48(1), 42-50.

Ruiz, J., Ortega, F., Castillo, R., Martín-Matillas, M., Kwak, L., Vicente-Rodríguez, G,... Moreno, L. (2010). Physical activity, fitness, weight status, and cognitive performance in adolescents. Journal of Pediatrics, 157(6), 917-922.

Rudolph, K. D. (2002). Gender differences in emotional responses to interpersonal stress during adolescence. Journal of Adolescent Health, 30(4), 3-13.

Sardinha, L., Marques, A., Martins, S., Palmeira, A., \& Minderico, C. (2014). Fitness, fatness, and academic performance in seventh grade elementary school students. BMC Pediatrics, 14, 176.

Segura-Díaz, J.M., Herrador-Colmenero, M., Martínez-Téllez, B., \& Chillón, P. (2015). Efecto de la precipitación y el periodo estacional sobre los patrones de desplazamiento al centro educativo en niños y adolescentes de Granada. Nutrición Hospitalaria, 31(3), 1264-1272.

Smith, L., Sahlqvist, S., Ogilvie, D., Jones, A., Corder, K., Griffin, S. J., \& van Sluijs, E. (2012). Is a change in mode of travel to school associated with a change in overall physical activity levels in children? Longitudinal results from the SPEEDY study. International Journal of Behavioral Nutrition and Physical Activity, 9, 134.

So, W. (2012). Association between physical activity and academic performance in Korean adolescent students. BMC Public Health, 12(1), 258.

Stock, C., Bloomfield, K., Ejstrud, B., Vinther-Larsen, M., y Meijer, M., Grønbæk, M., \& Grittner, U. (2012). Are characteristics of the school district associated with active transportation to school in Danish adolescents? European Journal of Public Health, 22(3), 398-404.

Suhrcke, M., \& de Paz Nieves, C. (2011). The impact of health and health behaviours on educational outcomes in high-incomes countries: a review of the evidence, Copenhagen, WHOregional Office for Europe.

Valle, A., Cabanach, R., González-Pienda, J. A., Núñez, J. C., Rodríguez, S., \& Rosario, P. (2009). Perfiles motivacionales en estudiantes de secundaria: análisis diferencial en estrategias cognitivas, estrategias de autorregulación y rendimiento académico. Revista Mexicana de Psicología, 26(1), 113-124.

Van Dijk, M. L., De Groot, R. H., Van Acker, F., Savelberg, H. H., \& Kirschner, P.A. (2014).Active commuting to school, cognitive performance, and academic achievement: an observational study in Dutch adolescents using accelerometers. BMCPublic Health, 14, 799.

Villa-González, E., Ruiz, J. R., \& Chillón, P. (2015). Associations between Active Commuting to School and Health-Related Physical Fitness in Spanish SchoolAgedChildren:ACross-Sectional Study.International Journal of Environmental Research and Public Health, 12(9), 10362-73.

Villa-González,E., Ruiz, J., \& Chillón, P.(2016). Recomendaciones para implementar intervenciones de calidad de promoción del desplazamiento activo al colegio. Retos, (30), 159-161.

Wrann, C. D., White, J. P., Salogiannnis, J., Laznik-Bogoslavski, D., Wu, J., Ma, D., ... Spiegelman, B. M. (2013). Exercise Induces Hippocampal BDNF through a PGC-1á/FNDC5 Pathway. Cell Metabolism, 18(5), 649-659.

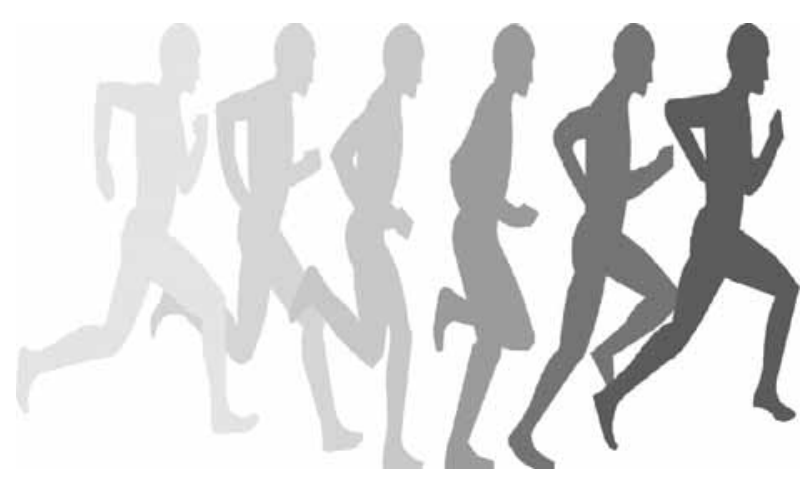

